\title{
Unitary Realizations of U-duality Groups as Conformal and Quasiconformal Groups and Extremal Black Holes of Supergravity Theories ${ }^{1}$
}

\author{
Murat Günaydin \\ Physics Department, Penn State University \\ University Park, PA 16802, USA \\ email:murat@phys.psu.edu
}

\begin{abstract}
We review the current status of the construction of unitary representations of Uduality groups of supergravity theories in five, four and three dimensions. We focus mainly on the maximal $N=8$ supergravity theories and on the $N=2$ MaxwellEinstein supergravity (MESGT) theories defined by Jordan algebras of degree three in five dimensions and their descendants in four and three dimensions. Entropies of the extremal black hole solutions of these theories in five and four dimensions are given by certain invariants of their U-duality groups. The five dimensional U-duality groups admit extensions to spectrum generating generalized conformal groups which are isomorphic to the U-duality groups of corresponding four dimensional theories. Similarly, the U-duality groups of four dimensional theories admit extensions to spectrum generating quasiconformal groups that are isomorphic to the corresponding Uduality groups in three dimensions. For example, the group $E_{8(8)}$ can be realized as a quasiconformal group in the 57 dimensional charge-entropy space of BPS black hole solutions of maximal $N=8$ supergravity in four dimensions and leaves invariant "lightlike separations" with respect to a quartic norm. Similarly $E_{7(7)}$ acts as a generalized conformal group in the 27 dimensional charge space of BPS black hole solutions in five dimensional $N=8$ supergravity and leaves invariant "lightlike separations" with respect to a cubic norm. For the exceptional $N=2$ Maxwell-Einstein supergravity theory the corresponding quasiconformal and conformal groups are $E_{8(-24)}$ and $E_{7(-25)}$, respectively. We outline the oscillator construction of the unitary representations of generalized conformal groups that admit positive energy representations, which include the U-duality groups of $N=2$ MESGT's in four dimensions . We conclude with a review of the minimal unitary realizations of U-duality groups that are obtained by quantizations of their quasiconformal actions and discuss in detail the minimal unitary realization of $E_{8(8)}$.
\end{abstract}

\footnotetext{
${ }^{1}$ Invited talk at the XIXth Max Born Symposium on Fundamental Interactions and Twistorlike Methods ( Wroclaw, Sept. 28 - Oct. 1, 2004). To appear in the proceedings of the symposium in the AIP proceedings series .
} 


\section{U-Duality Groups in Supergravity Theories}

\subsection{Noncompact exceptional groups as symmetries of maximally ex- tended supergravity theories in various dimensions}

Eleven dimensional supergravity [1] is the effective low energy theory of strongly coupled phase of M-theory [2] and its toroidal compactifications yield the maximally extended supergravity theories in $d$ spacetime dimensions with global non-compact symmetry groups of type $E_{(11-d)(11-d)}[3]$. The discrete subgroups $E_{(11-d)(11-d)}(\mathbb{Z})$ of these groups are expected to be the symmetries of the non-perturbative spectra of toroidally compactified Mtheory [4]. We shall use the term U-duality group to refer to these discrete subgroups as well as to the global noncompact symmetry groups of supergravity theories.

In five dimensions $E_{6(6)}$ is a symmetry of the Lagrangian of the maximal ( $N=8$ ) supergravity, under which the 27 vector fields of the theory transform irreducibly while the 42 scalar fields transform nonlinearly and parameterize the coset space

$$
\mathcal{M}_{5}=E_{6(6)} / U S p(8)
$$

On the other hand the $E_{7(7)}$ symmetry of the maximally extended supergravity in $d=4$ is an on-shell symmetry group. The 28 vector field strengths of this theory together with their "magnetic" duals ( 28 ) transform irreducibly in the 56 of $E_{7(7)}$ and 70 scalar fields parameterize the coset space

$$
\mathcal{M}_{4}=E_{7(7)} / S U(8)
$$

In three dimensions all the propagating bosonic degrees of the maximal $N=16$ supergravity can be dualized to scalar fields which parameterize the coset space

$$
\mathcal{M}_{3}=E_{8(8)} / S O(16)
$$

\subsection{U-duality groups in Matter coupled Supergravity Theories}

Non-compact global U-duality groups arise in matter coupled supergravity theories as well. In this talk I will focus mainly on U-duality groups in $N=2$ Maxwell-Einstein supergravity theories (MESGT) in $d=5$ and the corresponding theories in four and three dimensions. The MESGT's describe the coupling of an arbitrary number $n$ of (Abelian) vector fields to $N=2$ supergravity and five dimensional theories were constructed in [5]. The bosonic part of the Lagrangian can be written as [5] 


$$
\begin{aligned}
e^{-1} \mathcal{L}_{\text {bosonic }}= & -\frac{1}{2} R-\frac{1}{4} \stackrel{\circ}{a J}_{I J} F_{\mu \nu}^{I} F^{J \mu \nu}-\frac{1}{2} g_{x y}\left(\partial_{\mu} \varphi^{x}\right)\left(\partial^{\mu} \varphi^{y}\right) \\
& +\frac{e^{-1}}{6 \sqrt{6}} C_{I J K} \varepsilon^{\mu \nu \rho \sigma \lambda} F_{\mu \nu}^{I} F_{\rho \sigma}^{J} A_{\lambda}^{K},
\end{aligned}
$$

where $e$ and $R$ denote the fünfbein determinant and the scalar curvature in $d=5$, respectively. $F_{\mu \nu}^{I}$ are the field strengths of the Abelian vector fields $A_{\mu}^{I},(I=0,1,2 \cdots, n)$ with $A_{\mu}^{0}$ denoting the "bare" graviphoton. The metric, $g_{x y}$, of the scalar manifold $\mathcal{M}$ and the "metric" $\stackrel{\circ}{a}_{I J}$ of the kinetic energy term of the vector fields both depend on the scalar fields $\varphi^{x}(x, y, . .=1,2, . ., n)$. The invariance under Abelian gauge transformations of the vector fields requires the completely symmetric tensor $C_{I J K}$ to be constant. Remarkably, one finds that the entire $N=2, d=5$ MESGT is uniquely determined by the constant tensor $C_{I J K}$ [5]. In particular, the metrics of the kinetic energy terms of the vector and scalar fields are determined by $C_{I J K}$. More specifically, consider the cubic polynomial, $\mathcal{V}(h)$, in $(n+1)$ real variables $h^{I}(I=0,1, \ldots, n)$ defined by the $C_{I J K}$

$$
\mathcal{V}(h):=C_{I J K} h^{I} h^{J} h^{K} .
$$

Using this polynomial as a real " Kähler potential" for a metric, $a_{I J}$, in an $n+1$ dimensional ambient space with the coordinates $h^{I}$ :

$$
a_{I J}(h):=-\frac{1}{3} \frac{\partial}{\partial h^{I}} \frac{\partial}{\partial h^{J}} \ln \mathcal{V}(h) .
$$

one finds that the $n$-dimensional target space, $\mathcal{M}$, of the scalar fields $\varphi^{x}$ can be identified with the hypersurface [5]

$$
\mathcal{V}(h)=C_{I J K} h^{I} h^{J} h^{K}=1
$$

in this space. The metric $g_{x y}$ of the scalar manifold is simply the pull-back of (6) to $\mathcal{M}$ and the "metric" $\stackrel{\circ}{a}_{I J}(\varphi)$ of the kinetic energy term of the vector fields appearing in (4) is given by the componentwise restriction of $a_{I J}$ to $\mathcal{M}$ :

$$
\stackrel{\circ}{a}_{I J}(\varphi)=a_{I J} \mid \mathcal{V}=1
$$

The physical requirement of positivity of kinetic energy requires that $g_{x y}$ and $\stackrel{\circ}{a}_{I J}$ be positive definite metrics. This requirement induces constraints on the possible $C_{I J K}$, and in [5] it was shown that any $C_{I J K}$ that satisfy these constraints can be brought to the following form

$$
C_{000}=1, \quad C_{0 i j}=-\frac{1}{2} \delta_{i j}, \quad C_{00 i}=0,
$$


with the remaining coefficients $C_{i j k}(i, j, k=1,2, \ldots, n)$ being completely arbitrary. This basis is referred to as the canonical basis for $C_{I J K}$.

Denoting the symmetry group of the tensor $C_{I J K}$ as $G$ one finds that the full symmetry group of $N=2$ MESGT in $d=5$ is of the form

$$
G \times S U(2)_{R}
$$

where $S U(2)_{R}$ denotes the local R-symmetry group of the $N=2$ supersymmetry algebra. A MESGT is said to be unified if all the vector fields, including the graviphoton, transform in an irreducible representation of a simple symmetry group $G$ of the lagrangian. Of all the $N=2$ MESGT's whose scalar manifolds are symmetric spaces only four are unified [5]. More recently it was shown that if one relaxes the condition that the scalar manifolds be homogeneous spaces then one finds three novel infinite families ( plus an additional sporadic one ) of unified MESGT's in $d=5$ [6]. If one defines a cubic form

$$
\mathcal{N}(h):=C_{I J K} h^{I} h^{J} h^{K}
$$

using the constant tensor $C_{I J K}$, one finds that the cubic forms associated with the four unified MESGT's can be identified with the norm forms of simple ( Euclidean) Jordan algebras of degree three [5]. There exist only four simple ( Euclidean) Jordan algebras of degree three and they can be realized in terms of $3 \times 3$ hermitian matrices over the four division algebras with the product being one-half the anticommutator. They are denoted as $J_{3}^{\mathbb{A}}$, where $\mathbb{A}$ stands for the underlying division algebra, which can be real numbers $\mathbb{R}$, complex numbers $\mathbb{C}$, quaternions $\mathbb{H}$ and octonions $\mathbb{O}$. The supergravity theories defined by them were referred to as magical supergravity theories [5] since their symmetry groups in five, four and three dimensions correspond precisely to the symmetry groups of the famous Magic Square . The octonionic Jordan algebra $J_{3}^{\mathbb{D}}$ is the unique exceptional Jordan algebra and consequently the $N=2$ MESGT defined by it is called the exceptional supergravity theory [5]. In the table below we list the scalar manifolds $G / H$ of the magical supergravity theories in five, four and three dimensions, where $G$ is the global noncompact symmetry and $H$ is its maximal compact subgroup.

\begin{tabular}{|c|c|c|c|}
\hline$J$ & G/H in d=5 & G/H in d=4 & G/H in d=3 \\
\hline$J_{3}^{\mathbb{R}}$ & $S L(3, \mathbb{R}) / S O(3)$ & $S p(6, \mathbb{R}) / U(3)$ & $F_{4(4)} / U S p(6) \times S U(2)$ \\
\hline$J_{3}^{\mathbb{C}}$ & $S L(3, \mathbb{C}) / U(3)$ & $S U(3,3) / S U(3)^{2} \times U(1)$ & $E_{6(2)} / S U(6) \times S U(2)$ \\
\hline$J_{3}^{\mathbb{1 1}}$ & $S U^{*}(6) / U S p(6)$ & $S O^{*}(12) / U(6)$ & $E_{7(-5)} / S O(12) \times S U(2)$ \\
\hline$J_{3}^{\bigotimes}$ & $E_{6(-26)} / F_{4}$ & $E_{7(-25)} / E_{6} \times U(1)$ & $E_{8(-24)} / E_{7} \times S U(2)$ \\
\hline
\end{tabular}

Note that the exceptional $N=2$ supergravity has $E_{6(-26)}, E_{7(-25)}$ and $E_{8(-24)}$ as its global symmetry groups in five, four and three dimensions, respectively, whereas the maximally extended supergravity theory has the maximally split real forms $E_{6(6)}, E_{7(7)}$ and $E_{8(8)}$ as its symmetry groups in the corresponding dimensions. 
In addition to four simple Euclidean Jordan algebras $J_{3}^{\mathbb{A}}$ there exist an infinite family of nonsimple Jordan algebras of degree three, which are direct sums $J=\mathbb{R} \oplus \Gamma(Q)$ of a one dimensional Jordan algebra $\mathbb{R}$ with a Jordan algebra $\Gamma(Q)$ associated with a quadratic form $Q^{2}$. This family of $N=2$ MESGT's is called the generic Jordan family and their scalar manifolds in five dimensions are

$$
\mathcal{M}_{5}(\mathbb{R} \oplus \Gamma(Q))=\frac{S O(1,1) \times S O(n-1,1)}{S O(n)}
$$

and $S O(1,1) \times S O(n-1,1)$ are the global symmetry groups of their Lagrangians. The corresponding $N=2$ MESGT's in $d=4$ obtained by dimensional reduction have the scalar manifolds:

$$
\mathcal{M}_{4}(\mathbb{R} \oplus \Gamma(Q))=\frac{S O(2,1) \times S O(n, 2)}{S O(2) \times S O(n) \times S O(2)}
$$

By further reduction to three dimensions the scalar manifolds become

$$
\mathcal{M}_{3}\left((\mathbb{R} \oplus \Gamma(Q))=\frac{S O(n+2,4)}{S O(n+2) \times S O(4)}\right.
$$

The term U-duality was introduced by Hull and Townsend since the discrete symmetry group $E_{7(7)}(\mathbb{Z})$ of $\mathrm{M} /$ superstring theory toroidally compactified to $d=4$ unifies the Tduality group $S O(6,6)(\mathbb{Z})$ with the $S$-duality group $S L(2, \mathbb{Z})$ in a simple group since

$$
S O(6,6) \times S L(2, \mathbb{R}) \subset E_{7(7)}
$$

The analogous decomposition of the symmetry group $E_{7(-25)}$ of the exceptional supergravity in $d=4$ is

$$
S O(10,2) \times S L(2, \mathbb{R}) \subset E_{7(-25)}
$$

with similar decompositions for the other magical supergravity theories. For the generic Jordan family of $N=2$ MESGT's there is no simple U-duality group that unifies the corresponding $\mathrm{S}(S L(2, \mathbb{R}))$ and T-duality $(S O(n+2,2))$ groups.

\footnotetext{
${ }^{2}$ The positivity of the kinetic terms of scalars and vectors requires the metric of the quadratic form to me Minkowskian .
} 


\section{U-duality Groups and Entropy of BPS Black Holes in Supergravity Theories}

The entropy of BPS black hole solutions in maximally extended supergravity as well as in matter coupled supergravity theories are invariant under the corresponding U-duality groups. For example in $d=5, N=8$ supergravity the entropy $S$ of a BPS black hole solution can be written in the form [7]

$$
S=\alpha \sqrt{I_{3}}=\alpha \sqrt{d_{I J K} q^{I} q^{J} q^{K}}
$$

where $\alpha$ is some fixed constant and $I_{3}$ is the cubic invariant of $E_{6(6)}$ with the $q^{I}, I=$ $0,2, \ldots, 26$ denoting the charges coupling to 27 vector fields of the theory. The BPS black hole solutions with $I_{3} \neq 0$ preserve $1 / 8$ supersymmetry [8] and the solutions with $I_{3}=0$ , but with $\frac{1}{3} \partial_{I} I_{3}=d_{I J K} q^{J} q^{K} \neq 0$ preserve $1 / 4$ supersymmetry. The BPS black hole solutions with both $I_{3}=0$ and $d_{I J K} q^{J} q^{K}=0$ preserve $1 / 2$ supersymmetry [8]. The orbits of the BPS black hole solutions of $N=8$ supergravity in $d=5$ under the action of its U-duality group $E_{6(6)}$ were classified in [9].

The entropy of the BPS black hole solutions of five dimensional $N=2$ MESGT's is given by the cubic form defined by the constant tensor [7]

$$
S=\alpha \sqrt{\mathcal{N}}=\alpha \sqrt{C_{I J K} q^{I} q^{J} q^{K}}
$$

For those $N=2$ MESGT theories defined Jordan algebras of degree three this cubic form is the norm form and the global symmetry group $G$ is its invariance group as explained in the previous section. The orbits of the BPS black hole solutions of $5 d, N=2$ MESGT's defined by Jordan algebras under the actions of their U-duality groups were classified in [9]. This was achieved by associating with a given BPS solution with charges $q^{I},(I=0,1, \ldots n)$ an element

$$
J=\sum_{I=0}^{n} e_{I} q^{I}
$$

of the Jordan algebra of degree three, where $e_{I}$ form a basis of the Jordan algebra. This establishes a correspondence between the Jordan algebra and the charge space of the BPS black hole solutions.

Similarly, the classification of the orbits of BPS black hole solutions of the $N=8$ supergravity in $d=5$ as given in [9] associates with a given BPS black hole solution with charges $q^{I}$ an element $J=\sum_{I=0}^{26} e_{I} q^{I}$ of the split exceptional Jordan algebra with basis 
elements $e_{I}{ }^{3}$. The cubic invariant $I_{3}\left(q^{I}\right)$ is then simply given by the norm form $\mathcal{N}$ of the split exceptional Jordan algebra. Invariance group $E_{6(6)}$ of this norm form ( known as the reduced structure group in mathematics literature) is the U-duality group of the maximal $N=8$ supergravity theory in $d=5$.

In four dimensional magical $N=2$ MESGT's obtained by dimensional reduction from five dimensions as well as the maximal $4 d, N=8$ supergravity the entropies of BPS black hole solutions are given by the quartic invariants of their U-duality groups [10]

$$
S=\beta \sqrt{I_{4}}=\beta \sqrt{d_{I J K L} q^{I} q^{J} q^{K} q^{L}}
$$

where $d_{I J K L}$ are the completely symmetric tensors defined by the Freudenthal-Kantor triple systems associated with the corresponding simple Jordan algebras of degree three [9] and $q^{I}$ now denote both electric and magnetic charges. For the generic Jordan family of $N=2$ MESGT's in $d=4$ the quartic invariants are defined by the completely symmetric tensors of the Freudenthal-Kantor triple systems associated with the groups $S O(n+4,4)$. The orbits of the BPS black hole solutions of these theories under the action of the corresponding U-duality groups were given in [9]. For the maximal $N=8$ supergravity $q^{I}$ represent 28 electric and 28 magnetic charges. The number of supersymmetries preserved by the extremal black hole solutions of the $N=8$ theory depends on whether or not $I_{4}$, $\partial_{J} I_{4}$ and $\partial_{J} \partial_{K} I_{4}$ vanish [8].

\section{Generalized space-times defined by Jordan algebras}

\subsection{Generalized Rotation, Lorentz and Conformal Groups}

In the previous sections we saw how Jordan algebras arise in a fundamental way within the framework of supergravity theories. In this section I will review how Jordan algebras appear naturally within the framework of attempts to generalize four dimensional Minkowski spacetime and its symmetry groups. The first proposal to use Jordan algebras to define generalized spacetimes was made in the early days of spacetime supersymmetry while searching for the super analogs of the exceptional Lie algebras [11].

Now the twistor formalism in four-dimensional space-time $(d=4)$ leads naturally to the representation of four vectors in terms of $2 \times 2$ Hermitian matrices over the field of

\footnotetext{
${ }^{3}$ Split exceptional Jordan algebra $J_{3}^{\mathbb{Q}_{s}}$ is realized in terms of hermitian $3 \times 3$ matrices over the split octonions $\mathbb{O}_{s}$, which is not a division algebra. As a consequence $J_{3}^{\mathbb{O}_{s}}$ is not a Euclidean ( formally real) Jordan algebra in contrast to the real exceptional Jordan algebra $J_{3}^{\mathbb{Q}}$ of the exceptional $N=2$ theory, which is defined over the division algebra of real octonions $\mathbb{O}$. A Jordan algebra $J$ is called Euclidean if for any pair of elements $X, Y \in J$ the equation $X^{2}+Y^{2}=0$ implies that $X=0$ and $Y=0$.
} 
complex numbers $\mathbb{C}$. In particular, the coordinate four vectors $x_{\mu}$ can be represented as :

$$
x=x_{\mu} \sigma^{\mu}
$$

Since the Hermitian matrices over the field of complex numbers close under the symmetric anti-commutator product one can regard the coordinate vectors as elements of a Jordan algebra denoted as $J_{2}^{\mathbb{C}}[11,12]$. Then the rotation, Lorentz and conformal groups in $d=4$ can be identified with the automorphism, reduced structure and Möbius ( linear fractional) groups of the Jordan algebra of $2 \times 2$ complex Hermitian matrices $J_{2}^{\mathbb{C}}[11,12]$. The reduced structure group $\operatorname{Str}_{0}(J)$ of a Jordan algebra $J$ is simply the invariance group of its norm form $N(J)$. (The structure group $\operatorname{Str}(J)=\operatorname{Str}_{0}(J) \times S O(1,1)$,on the other hand, is simply the invariance group of $N(J)$ up to an overall constant scale factor.) Furthermore, this interpretation leads one naturally to define generalized space-times whose coordinates are parameterized by the elements of Jordan algebras [11]. The rotation $\operatorname{Rot}(J)$, Lorentz $\operatorname{Lor}(J)$ and conformal $C o n(J)$ groups of these generalized space-times are then identified with the automorphism $A u t(J)$, reduced structure $\operatorname{Str}_{0}(J)$ and Möbius Mö(J) groups of the corresponding Jordan algebra [11, 12, 13, 14]. Denoting as $J_{n}^{\mathbb{A}}$ the Jordan algebra of $n \times n$ Hermitian matrices over the division algebra $\mathbb{A}$ and the Jordan algebra of Dirac gamma matrices in $d$ (Euclidean) dimensions as $\Gamma(d)$ one finds the following symmetry groups of generalized space-times defined by simple Euclidean ( formally real) Jordan algebras:

\begin{tabular}{|c|c|c|c|}
\hline$J$ & Rotation $(J)$ & Lorentz $(J)$ & Conformal $(J)$ \\
\hline$J_{n}^{\mathbb{R}}$ & $S O(n)$ & $S L(n, \mathbb{R})$ & $S p(2 n, \mathbb{R})$ \\
$J_{n}^{\mathbb{C}}$ & $S U(n)$ & $S L(n, \mathbb{C})$ & $S U(n, n)$ \\
$J_{n}^{\mathbb{H}}$ & $U S p(2 n)$ & $S U^{*}(2 n)$ & $S O^{*}(4 n)$ \\
$J_{3}^{\mathbb{O}}$ & $F_{4}$ & $E_{6(-26)}$ & $E_{7(-25)}$ \\
$\Gamma(d)$ & $S O(d)$ & $S O(d, 1)$ & $S O(d, 2)$ \\
\hline
\end{tabular}

The symbols $\mathbb{R}, \mathbb{C}, \mathbb{H}, \mathbb{O}$ represent the four division algebras. For the Jordan algebras $J_{n}^{\mathbb{A}}$ the norm form is the determinental form ( or its generalization to the quaternionic and octonionic matrices). For the Jordan algebra $\Gamma(d)$ generated by Dirac gamma matrices $\Gamma_{i}(i=1,2, \ldots d)$

$$
\left\{\Gamma_{i}, \Gamma_{j}\right\}=\delta_{i j} \mathbf{1} ; \quad i, j, \ldots=1,2, \ldots, d
$$


the norm of a general element $x=x_{0} \mathbf{1}+x_{i} \Gamma_{i}$ of $\Gamma(d)$ is quadratic and given by

$$
N(x)=x \bar{x}=x_{0}^{2}-x_{i} x_{i}
$$

where $\bar{x}=x_{0} \mathbf{1}-x_{i} \Gamma_{i}$. Its automorphism, reduced structure and Möbius groups are simply the rotation, Lorentz and conformal groups of $(d+1)$-dimensional Minkowski spacetime. One finds the following special isomorphisms between the Jordan algebras of $2 \times 2$ Hermitian matrices over the four division algebras and the Jordan algebras of gamma matrices:

$$
J_{2}^{\mathbb{R}} \simeq \Gamma(2) \quad ; \quad J_{2}^{\mathbb{C}} \simeq \Gamma(3) \quad ; \quad J_{2}^{\mathbb{H}} \simeq \Gamma(5) \quad ; \quad J_{2}^{\mathbb{Q}} \simeq \Gamma(9)
$$

The Minkowski spacetimes they correspond to are precisely the critical dimensions for the existence of super Yang-Mills theories as well as of the classical Green-Schwarz superstrings. These Jordan algebras are all quadratic and their norm forms are precisely the quadratic invariants constructed using the Minkowski metric.

We should note two remarkable facts about the above table. First, the conformal groups of generalized space-times defined by Euclidean ( formally real) Jordan algebras all admit positive energy unitary representations ${ }^{4}$. Hence they can be given a causal structure with a unitary time evolution as in four dimensional Minkowski space-time. Second is the fact that the maximal compact subgroups of the generalized conformal groups of formally real Jordan algebras are simply the compact forms of their structure groups (which are the products of their generalized Lorentz groups with dilatations), whose importance will be explained in the next subsection.

\subsection{Covariant Quantum Fields over Generalized Spacetimes and the Positive Energy Unitary Representations of Their Conformal Groups}

The Lie algebra $g$ of a non-compact group $\mathrm{G}$ that admits unitary lowest weight representations (ULWR) ( positive energy ) has a 3-grading with respect to the Lie algebra $h$ of its maximal compact subgroup $\mathrm{H}$ i.e

$$
g=g^{-1} \oplus g^{0} \oplus g^{+1}
$$

\footnotetext{
${ }^{4}$ Similarly, the generalized conformal groups defined by Hermitian Jordan triple systems all admit positive energy unitary representations [14]. In fact the conformal groups of simple Hermitian Jordan triple systems exhaust the list of simple noncompact groups that admit positive energy unitary representations. They include the conformal groups of simple Euclidean Jordan algebra since the latter form an hermitian Jordan triple system under the Jordan triple product [14].
} 
where $g^{0}=h$ and we have the formal commutation relations

$$
\left[g^{(m)}, g^{(n)}\right] \subseteq g^{(m+n)} \quad m, n=\mp 1,0
$$

and $g^{(m)} \equiv 0$ for $|m|>1$.

In [15] the general oscillator construction of unitary lowest weight representations of non-compact groups was given. To construct the ULWR's of a noncompact group $G$ one realizes its generators in terms of bilinears of bosonic oscillators transforming in a certain representation of $H$. Then in the corresponding Fock space $\mathcal{F}$ of all the oscillators one chooses a set of states $\mid \Omega>$, referred to as the "lowest weight vector" (lwv), which transforms irreducibly under $H$ and which are annihilated by the generators belonging to the $g^{-1}$ space. Then by acting on $\mid \Omega>$ repeatedly with the generators belonging to the $g^{+1}$ space one obtains an infinite set of states

$$
\left|\Omega>, \quad g^{+1}\right| \Omega>, \quad g^{+1} g^{+1} \mid \Omega>, \ldots
$$

that form the basis of an irreducible unitary lowest weight representation of $g$. (The irreducibility of the representation of $g$ follows from the irreducibility of the lwv $\mid \Omega>$ under $h$. We shall refer to this basis as the compact "particle" basis.

For example, the conformal group of the Jordan algebra $J_{2}^{\mathbb{C}}$ corresponding to the four dimensional Minkowski space is $S U(2,2)$ with a maximal compact subgroup $S U(2) \times$ $S U(2) \times U(1)$ which is simply the compact form of the structure group $S L(2, \mathbb{C}) \times$ $S O(1,1)$. In [17] it was explicitly shown how to go from the compact $S U(2) \times S U(2) \times$ $U(1)$ basis of the ULWR's of $S U(2,2)$ to the manifestly covariant $S L(2, \mathbb{C}) \times S O(1,1)$ basis. The transition from the compact to the covariant basis corresponds simply to going from a compact "particle" basis to a noncompact coherent state basis of the ULWR. The noncompact coherent states are labelled by the elements of $J_{2}^{\mathbb{C}}$ i.e by the coordinates of four dimensional Minkowski space. One thereby establishes a one-to-one correspondence between irreducible ULWR's of $S U(2,2)$ and the fields transforming irreducibly under the Lorentz group $S L(2, \mathbb{C})$ with a definite conformal dimension. Thus one can associate with irreducible ULWR's of $S U(2,2)$ conformal fields transforming covariantly under the Lorentz group with a definite conformal dimension.

Similarly, the conformal group $S O^{*}(8)$ of the Jordan algebra $J_{2}^{\mathbb{H}}$ parameterizing the six dimensional Minkowski space has a maximal compact subgroup $U(4)$ which is the compact form of the structure group $S U^{*}(4) \times S O(1,1)$. In [18] it was shown explicitly how to go from the compact $U(4)$ particle basis of the ULWR's of $S O^{*}(8)$ to the non-compact $S U^{*}(4) \times S O(1,1)$ coherent state basis. $\left(S U^{*}(4) \times S O(1,1)\right.$ is simply the covering group of the Lorentz group in six dimensions times dilatations). The coherent states of the noncompact basis are again labelled by the elements of $J_{2}^{\mathbb{H}}$, i.e the coordinates of $6 \mathrm{~d}$ Minkowski 
space. Thus each irreducible ULWR of $S O^{*}(8)$ can be identified with a field transforming covariantly under the Lorentz group $S U^{*}(4)$ with a definite conformal dimension.

The results obtained explicitly for the conformal groups of $J_{2}^{\mathbb{C}}$ and $J_{2}^{\mathbb{H}}$ extend to the conformal groups of all formally real Jordan algebras and of Hermitian Jordan triple systems [16, 19]. The general theory can be summarized as follows: Let $g$ be the Lie algebra of the conformal group of a formally real Jordan algebra and $g^{0}$ the Lie algebra of its maximal compact subgroup. Then $g$ has a three-graded decomposition with respect to $g^{0}$ :

$$
g=g^{-}+g^{0}+g^{+}
$$

where the grading is determined by the "conformal energy operator". Now let $n^{0}$ be the Lie algebra of the structure group ( Lorentz group times dilatations ) of the Euclidean Jordan algebra ( or of a Hermitian Jordan triple system). As stated above the Lie algebra $g$ has a 3-graded decomposition with respect to $n^{0}$ as well:

$$
g=n^{-}+n^{0}+n^{+}
$$

where the grading is defined by the generator of scale transformations. In the compact basis an irreducible ULWR of $\operatorname{Conf}(J)$ is uniquely determined by a lowest weight vector $|\Omega\rangle$ transforming irreducibly under the maximal compact subgroup $K$ that is annihilated by the operators belonging to $g^{-}$

$$
g^{-}|\Omega\rangle=0
$$

As was done explicitly for the conformal groups in 4 and 6 dimensions [17, 18] one can show that there exists a rotation operator $W$ in the representation space with the property that the states $W|\Omega\rangle$ are annihilated by all the generators belonging to $n^{-}$

$$
n^{-} W|\Omega\rangle=0
$$

and transform in a finite dimensional non-unitary representation of the non-compact structure group. Remarkably the transformation properties of $W|\Omega\rangle$ under the structure group coincide with the transformation properties of $|\Omega\rangle$ under the maximal compact subgroup $K$. In particular, the conformal dimension of the vector $W|\Omega\rangle$ is simply the negative of the conformal energy of $|\Omega\rangle$. If one chooses a basis $e_{\mu}$ for the Jordan algebra $J$ and denote the generators of generalized translations in the space $n^{+}$corresponding to $e_{\mu}$ as $P_{\mu}$, then the noncompact coherent states defined by the action of generalized translations on $W|\Omega\rangle$

$$
\left.\left|\Phi\left(x_{\mu}\right\rangle:=e^{i x^{\mu} P_{\mu}} W\right| \Omega\right\rangle
$$


form the covariant basis of the ULWR of the generalized conformal group $\operatorname{Con}(J)^{5}$. The coherent states $\mid \Phi\left(x_{\mu}\right\rangle$ labelled by the coordinates correspond to conformal fields transforming covariantly under the Lorentz group with a definite conformal dimension. Since the state $W|\Omega\rangle$ is annihilated by the generators of special conformal transformations $K_{\mu}$ belonging to the space $n^{-}$this proves that the irreducible ULWR's are equivalent to representations induced by finite dimensional irreps of the Lorentz group with a definite conformal dimension and trivial special conformal transformation properties. This generalizes the well-known construction of the positive energy representations of the four dimensional conformal group $S U(2,2)$ [21] to all generalized conformal groups of formally real Jordan algebras and Hermitian Jordan triple systems. They are simply induced representations with respect to the maximal parabolic subgroup $\operatorname{Str}(J) \odot S_{J}$ where $\odot$ denotes semi-direct product and $S_{J}$ is the Abelian subgroup generated by generalized special conformal transformations.

We should perhaps note that the generalized Poincare groups associated with the spacetimes defined by Jordan algebras are of the form

$$
\mathcal{P G}(J):=\operatorname{Lor}(J) \odot T_{J}
$$

where $T_{J}$ is the Abelian subgroup generated by generalized translations $P_{\mu}$. For quadratic Jordan algebras, $\Gamma(d), \mathcal{P} \mathcal{G}(\Gamma(d))$ is simply the Poincaré group in $d$ dimensional Minkowski space. The group $\mathcal{P} \mathcal{G}(\Gamma(d))$ has a quadratic Casimir operator $M^{2}=P_{\mu} P^{\mu}$ which is simply the mass operator. For Jordan algebras $J$ of degree $n$ the generalized Poincaré group $\mathcal{P} \mathcal{G}(J)$ has a Casimir invariant of order $n$ constructed out of the generalized translation generators $P_{\mu}$. For example for the real exceptional Jordan algebra $J_{3}^{\mathbb{Q}}$ the corresponding Casimir invariant is cubic and has the form

$$
M^{3}=C_{\mu \nu \rho} P^{\mu} P^{\nu} P^{\rho}
$$

where $C_{\mu \nu \rho}$ is the symmetric invariant tensor of the generalized Lorentz group $E_{6(-26)}$ of $J_{3}^{\mathbb{Q}}(\mu, \nu, \rho, . .=0,1, \ldots 26)^{6}$.

\footnotetext{
${ }^{5}$ We should note that the (super) coherent states associated with ULWR's of non-compact (super) groups introduced in [20] are labelled by complex (super) "coordinates" in the compact basis. These (super) coordinates parametrize the (super) hermitian symmetric space $G / H$.

${ }^{6}$ We should note that we are relabelling the indices $I, J, .$. with Greek characters $\mu, \nu, \ldots$ so as to emphasize the fact that ,in this section, we are looking at Jordan algebras from a spacetime point of view.
} 


\section{Conformal and quasi-conformal extensions of U-duality groups in five and four dimensions}

As discussed above we can associate an element $J=\sum_{I=0}^{26} e_{I} q^{I}$ of the real ( split) exceptional Jordan algebra $J_{3}^{\mathbb{Q}}\left(J_{3}^{\mathbb{Q}_{s}}\right)$ with a BPS black hole solution of the exceptional $N=2(N=8)$ supergravity theory with charges $q^{I}$ in five dimensions. Its entropy $S$ is given by the square root of the cubic norm $\mathcal{N}(J)$ of $J$, whose invariance group is $E_{6(-26)}($ $\left.E_{6(6)}\right)$.

Acting on an element $J=\sum_{I=0}^{26} e_{I} q^{I}$ of $J_{3}^{\mathbb{Q}}\left(J_{3}^{\mathbb{Q}_{s}}\right)$ by its conformal group $E_{7(-25)}\left(E_{7(7)}\right)$ changes its norm and hence the corresponding entropy. Thus one can regard $E_{7(-25)}\left(E_{7(7)}\right)$ as a spectrum generating symmetry in the charge space of BPS black hole solutions of the exceptional $N=2(N=8)$ supergravity in five dimensions [22, 23, 26]. If one defines a distance function between any two solutions with charges $q^{I}$ and $q^{I I}$ as the cubic norm of their difference

$$
d\left(q, q^{\prime}\right) \equiv \mathcal{N}_{3}\left(J-J^{\prime}\right)
$$

one finds that the light like separations are preserved under the conformal action of $E_{7(-25)}\left(E_{7(7)}\right)$ [22, 26]. The explicit action of $E_{7(7)}$ and $E_{7(-25)}$ on the corresponding 27 dimensional spaces are given in [22] and [26], respectively. Let us review briefly the conformal action of $E_{7(7)}$ given in [22]. Lie algebra of $E_{7(7)}$ has a 3-graded decomposition

$$
133=27 \oplus(78 \oplus \mathbf{1}) \oplus \overline{\mathbf{2 7}}
$$

under its $E_{6(6)} \times \mathcal{D}$ subgroup, where $\mathcal{D}$ represents the dilatation group $S O(1,1)$. Under its maximal compact subalgebra USp $(8)$ Lie algebra $E_{6(6)}$ decomposes as a symmetric tensor $\tilde{G}^{i j}$ in the adjoint 36 of $\operatorname{USp}(8)$ and a fully antisymmetric symplectic traceless tensor $\tilde{G}^{i j k l}$ transforming as the 42 of $\operatorname{USp}(8)$ (indices $1 \leq i, j, \ldots \leq 8$ are $\operatorname{USp}(8)$ indices). $\tilde{G}^{i j k l}$ is traceless with respect to the real symplectic metric $\Omega_{i j}=-\Omega_{j i}=-\Omega^{i j}$ (thus $\Omega_{i k} \Omega^{k j}=\delta_{i}^{j}$ ). The symplectic metric is used to raise and lower indices, with the convention that this is always to be done from the left. The other generators of conformal $E_{7(7)}$ consist of a dilatation generator $\tilde{H}$, translation generators $\tilde{E}^{i j}$ and the nonlinearly realized "special conformal" generators $\tilde{F}^{i j}$, transforming as 27 and $\overline{\mathbf{2 7}}$, respectively.

The fundamental 27 of $E_{6(6)}$ on which $\left.E_{7(7)}\right)$ acts nonlinearly can be represented as the symplectic traceless antisymmetric tensor $\tilde{Z}^{i j}$ transforming as ${ }^{7}$

\footnotetext{
${ }^{7}$ Throughout we use the convention that indices connected by a bracket are antisymmetrized with weight one.
} 


$$
\begin{aligned}
\tilde{G}_{j}^{i}\left(\tilde{Z}^{k l}\right) & =2 \delta_{j}^{k \tilde{Z}^{i l}}, \\
\tilde{G}^{i j k l}\left(\tilde{Z}^{m n}\right) & =\frac{1}{24} \epsilon^{i j k l m n p q} \tilde{Z}_{p q},
\end{aligned}
$$

where $\tilde{Z}_{i j}:=\Omega_{i k} \Omega_{j l} \tilde{Z}^{k l}=\left(\tilde{Z}^{i j}\right)^{*} \quad$ and $\quad \Omega_{i j} \tilde{Z}^{i j}=0$. The conjugate $\overline{\mathbf{2 7}}$ representation transforms as

$$
\begin{aligned}
\tilde{G}^{i}{ }_{j}\left(\bar{Z}^{k l}\right) & =2 \delta_{j}^{\bar{k} \bar{Z}^{i l}}, \\
\tilde{G}^{i j k l}\left(\bar{Z}^{m n}\right) & =-\frac{1}{24} \epsilon^{i j k l m n p q} \bar{Z}_{p q} .
\end{aligned}
$$

The cubic invariant of $E_{6(6)}$ in the 27 is given by

$$
\mathcal{N}_{3}(\tilde{Z}):=\tilde{Z}^{i j} \tilde{Z}_{j k} \tilde{Z}^{k l} \Omega_{i l}
$$

The generators $\tilde{E}^{i j}$ act as translations on the space with coordinates $\tilde{Z}^{i j}$ as :

$$
\tilde{E}^{i j}\left(\tilde{Z}^{k l}\right)=-\Omega^{i[k} \Omega^{l] j}-\frac{1}{8} \Omega^{i j} \Omega^{k l}
$$

and $\tilde{H}$ by dilatations

$$
\tilde{H}\left(\tilde{Z}^{i j}\right)=\tilde{Z}^{i j}
$$

The " special conformal generators" $\tilde{F}^{i j}$ in the $\overline{\mathbf{2 7}}$ are realized nonlinearly:

$$
\begin{aligned}
\tilde{F}^{i j}\left(\tilde{Z}^{k l}\right):= & -2 \tilde{Z}^{i j}\left(\tilde{Z}^{k l}\right)+\Omega^{i[k} \Omega^{l] j}\left(\tilde{Z}^{m n} \tilde{Z}_{m n}\right)+\frac{1}{8} \Omega^{i j} \Omega^{k l}\left(\tilde{Z}^{m n} \tilde{Z}_{m n}\right) \\
& +8 \tilde{Z}^{k m} \tilde{Z}_{m n} \Omega^{n[i} \Omega^{j] l}-\Omega^{k l}\left(\tilde{Z}^{i m} \Omega_{m n} \tilde{Z}^{n j}\right)
\end{aligned}
$$

The norm form needed to define the $E_{7(7)}$ invariant "light cones" is constructed from the cubic invariant of $E_{6(6)}$. If we define the "distance" between $\tilde{X}$ and $\tilde{Y}$ as $\mathcal{N}_{3}(\tilde{X}-\tilde{Y})$ then it is manifestly invariant under $E_{6(6)}$ and under the translations $\tilde{E}^{i j}$. Under $\tilde{H}$ it transforms by a constant factor, whereas under the action of $\tilde{F}^{i j}$ one finds

$$
\tilde{F}^{i j}\left(\mathcal{N}_{3}(\tilde{X}-\tilde{Y})\right)=\left(\tilde{X}^{i j}+\tilde{Y}^{i j}\right) \mathcal{N}(\tilde{X}-\tilde{Y}) .
$$

which proves that the light cone in $\mathbb{R}^{27}$ with base point $\tilde{Y}$ defined by

$$
\mathcal{N}_{3}(\tilde{X}-\tilde{Y})=0
$$


is indeed invariant under $E_{7(7)}$.

The above formulas carry over in a straightforward manner to the conformal realization of $E_{7(-25)}$ on a 27 dimensional space coordinatized by the real exceptional Jordan algebra $J_{3}^{\complement}$. In this case the cubic form is invariant under $E_{6(-26)}$ which has $U S p(6,2)$ as a subgroup. The $U S p(8)$ covariant formulas above for $E_{7(7)}$ are then replaced by $U S p(6,2)$ covariant formulas [26, 27]. For the generic Jordan family of $N=2$ MESGT's in five dimensions the conformal extensions of U-duality groups $S O(1,1) \times S O(n-1,1)$ are the groups

$$
S O(2,1) \times S O(n, 2)
$$

which act as spectrum generating symmetry groups in $d=5$. These conformal groups of generic $d=5$ MESGT's as well as the conformal groups $E_{7(7)}$ and $E_{7(-25)}$ acting on the 27 dimensional charge spaces of the $N=8$ and the exceptional $N=2$ supergravity in five dimensions are isomorphic to the U-duality groups of the corresponding four dimensional theories obtained by dimensional reduction. We should stress the obvious fact that the conformal extensions of U-duality groups act nonlinearly on the charge space of five dimensional theories, whereas the isomorphic four dimensional U-duality groups act linearly on the charge space spanned by both electric and magnetic charges.

One may wander whether there exist "conformal extensions " of the four dimensional U-duality groups that act nonlinearly on the $4 \mathrm{~d}$ charge spaces as spectrum generating symmetry groups and are isomorphic to the U-duality groups of the corresponding three dimensional theories obtained by dimensional reduction. This question was investigated in [22] and it was shown there that in the case of maximal supergravity, even though there is no conformal action of $E_{8(8)}$, which is the corresponding U-duality group in $d=3$, it has a quasi-conformal group action on a 57 dimensional space which is an extension of the 56 dimensional charge space by an extra coordinate. For BPS black hole solutions in $d=4$ this extra coordinate can be taken to be the entropy [22].

The realization of quasi-conformal action of $E_{8(8)}$ uses the 5-graded decomposition of its Lie algebra with respect to the Lie algebra of its $E_{7(7)} \times \mathcal{D}$ subgroup

$$
\begin{gathered}
\mathfrak{g}^{-2} \oplus \mathfrak{g}^{-1} \oplus \quad \mathfrak{g}^{0} \quad \oplus \mathfrak{g}^{+1} \oplus \mathfrak{g}^{+2} \\
\mathbf{1} \oplus \mathbf{5 6} \oplus(\mathbf{1 3 3} \oplus \mathbf{1}) \oplus \mathbf{5 6} \oplus \mathbf{1}
\end{gathered}
$$

with $\mathcal{D}$ representing dilatations, whose generator together with grade \pm 2 elements generate an $S L(2, \mathbb{R})$ subgroup. It turns out to be very convenient to work in a basis covariant with respect to the $\operatorname{SL}(8, \mathbb{R})$ subgroup of $E_{7(7)}$ [22]. Let us denote the $\operatorname{SL}(8, \mathbb{R})$ covariant generators belonging to the grade $-2,-1,0,1$ and 2 subspaces in the above decomposition 
as follows:

$$
E \oplus\left\{E^{i j}, E_{i j}\right\} \oplus\left\{G^{i j k l}, G^{i}{ }_{j} ; H\right\} \oplus\left\{F^{i j}, F_{i j}\right\} \oplus F
$$

where $i, j, . .=1,2 \ldots, 8$ are now $\operatorname{SL}(8, \mathbb{R})$ indices.

Consider now a 57 -dimensional real vector space with coordinates

$$
\mathcal{X}:=\left(X^{i j}, X_{i j}, x\right)
$$

where $X^{i j}$ and $X_{i j}$ transform in the 28 and $\tilde{28}$ of $\operatorname{SL}(8, \mathbb{R})$ and $x$ is a singlet. The generators of $E_{7(7)}$ subalgebra act linearly on this space

$$
\begin{aligned}
& G^{i}{ }_{j}\left(X^{k l}\right)=2 \delta_{j}^{k} X^{i l}-\frac{1}{4} \delta_{j}^{i} X^{k l}, \quad G^{i j k l}\left(X^{m n}\right)=\frac{1}{24} \epsilon^{i j k l m n p q} X_{p q}, \\
& G^{i}{ }_{j}\left(X_{k l}\right)=-2 \delta_{k}^{i} X_{j l}+\frac{1}{4} \delta_{j}^{i} X_{k l}, G^{i j k l}\left(X_{m n}\right)=\delta_{m n}^{[i j} X^{k l]}, \\
& G^{i}{ }_{j}(x)=0, \quad G^{i j k l}(x)=0,
\end{aligned}
$$

The generator $H$ of dilatations acts as

$$
H\left(X^{i j}\right)=X^{i j}, \quad H\left(X_{i j}\right)=X_{i j}, \quad H(x)=2 x,
$$

and the generator $E$ acts as translations on $x$ :

$$
E\left(X^{i j}\right)=0, \quad E\left(X_{i j}\right)=0, \quad E(x)=1 .
$$

The grade \pm 1 generators act as

$$
\begin{aligned}
& E^{i j}\left(X^{k l}\right)=0, \quad E^{i j}\left(X_{k l}\right)=\delta_{k l}^{i j}, \quad E^{i j}(x)=-X^{i j}, \\
& E_{i j}\left(X^{k l}\right)=\delta_{i j}^{k l}, \quad E_{i j}\left(X_{k l}\right)=0, \quad E_{i j}(x)=X_{i j} .
\end{aligned}
$$

The positive grade generators are realized nonlinearly. The generator $F$ acts as

$$
\begin{aligned}
& F\left(X^{i j}\right)=4 X^{i k} X_{k l} X^{l j}+X^{i j} X^{k l} X_{k l} \\
& -\frac{1}{12} \epsilon^{i j k l m n p q} X_{k l} X_{m n} X_{p q}+X^{i j} x \\
& F\left(X_{i j}\right)=-4 X_{i k} X^{k l} X_{l j}-X_{i j} X^{k l} X_{k l} \\
& +\frac{1}{12} \epsilon_{i j k l m n p q} X^{k l} X^{m n} X^{p q}+X_{i j} x, \\
& F(x)=4 \mathcal{I}_{4}\left(X^{i j}, X_{i j}\right)+x^{2}
\end{aligned}
$$


where $\mathcal{I}_{4}$ is the quartic invariant of $E_{7(7)}$

$$
\begin{aligned}
\mathcal{I}_{4} \equiv X^{i j} X_{j k} X^{k l} X_{l i} & -\frac{1}{4} X^{i j} X_{i j} X^{k l} X_{k l}+\frac{1}{96} \epsilon^{i j k l m n p q} X_{i j} X_{k l} X_{m n} X_{p q} \\
& +\frac{1}{96} \epsilon_{i j k l m n p q} X^{i j} X^{k l} X^{m n} X^{p q}
\end{aligned}
$$

The action of the remaining generators of $E_{8(8)}$ are as follows:

$$
\begin{aligned}
& F^{i j}\left(X^{k l}\right)=-4 X^{i\left[k X^{l] j}\right.}+\frac{1}{4} \epsilon^{i j k l m n p q} X_{m n} X_{p q}, \\
& F^{i j}\left(X_{k l}\right)=+8 \delta_{k}^{[i} X^{j] m} X_{m l}+\delta_{k l}^{i j} X^{m n} X_{m n}+2 X^{i j} X_{k l}-\delta_{k l}^{i j} x, \\
& F_{i j}\left(X^{k l}\right)=-8 \delta_{[i}^{k X_{j] m} X^{m l}}+\delta_{i j}^{k l} X^{m n} X_{m n}-2 X_{i j} X^{k l}-\delta_{i j}^{k l} x, \\
& F_{i j}\left(X_{k l}\right)=4 X_{k i} X_{j l}-\frac{1}{4} \epsilon_{i j k l m n p q} X^{m n} X^{p q}, \\
& F^{i j}(x)=4 X^{i k} X_{k l} X^{l j}+X^{i j} X^{k l} X_{k l} \\
& -\frac{1}{12} \epsilon^{i j k l m n p q} X_{k l} X_{m n} X_{p q}+X^{i j} x \\
& F_{i j}(x)=4 X_{i k} X^{k l} X_{l j}+X_{i j} X^{k l} X_{k l} \\
& -\frac{1}{12} \epsilon_{i j k l m n p q} X^{k l} X^{m n} X^{p q}-X_{i j} x \text {. }
\end{aligned}
$$

The above action of $E_{8(8)}$ was called quasiconformal in [22] since it leaves a certain norm invariant up to an overall factor. Since the standard difference $(\mathcal{X}-\mathcal{Y})$ of two vectors in the 57 dimensional space is not invariant under "translations" generated by $\left(E^{i j}, E_{i j}\right)$, one defines a nonlinear difference that is invariant under these translations as:

$$
\delta(\mathcal{X}, \mathcal{Y}):=\left(X^{i j}-Y^{i j}, X_{i j}-Y_{i j} ; x-y+\langle X, Y\rangle\right)=-\delta(\mathcal{Y}, \mathcal{X})
$$

where $\langle X, Y\rangle:=X^{i j} Y_{i j}-X_{i j} Y^{i j}$. One defines the norm of a vector $\mathcal{X}$ in the 57 dimensional space as

$$
\mathcal{N}_{4}(\mathcal{X}) \equiv \mathcal{N}_{4}\left(X^{i j}, X_{i j} ; x\right):=\mathcal{I}_{4}(X)-x^{2},
$$

Then the "distance" between any two vectors $\mathcal{X}$ and $\mathcal{Y}$ defined as $\mathcal{N}_{4}(\delta(\mathcal{X}, \mathcal{Y}))$ is invariant under $E_{7(7)}$ and translations generated by $E^{i j}, E_{i j}$ and $E$. Under the action of the remaining generators of $E_{8(8)}$ one finds that

$$
\begin{aligned}
F\left(\mathcal{N}_{4}(\delta(\mathcal{X}, \mathcal{Y}))\right) & =2(x+y) \mathcal{N}_{4}(\delta(\mathcal{X}, \mathcal{Y})) \\
F^{i j}\left(\mathcal{N}_{4}(\delta(\mathcal{X}, \mathcal{Y}))\right) & =2\left(X^{i j}+Y^{i j}\right) \mathcal{N}_{4}(\delta(\mathcal{X}, \mathcal{Y})) \\
H\left(\mathcal{N}_{4}(\delta(\mathcal{X}, \mathcal{Y}))\right) & =4 \mathcal{N}_{4}(\delta(\mathcal{X}, \mathcal{Y}))
\end{aligned}
$$


Therefore, for every $\mathcal{Y} \in \mathbb{R}^{57}$ the "light cone" with base point $\mathcal{Y}$, defined by the set of $\mathcal{X} \in \mathbb{R}^{57}$ satisfying

$$
\mathcal{N}_{4}(\delta(\mathcal{X}, \mathcal{Y}))=0
$$

is preserved by the full $E_{8(8)}$ group.

The quasiconformal realization of the other real noncompact form $E_{8(-24)}$ with the maximal compact subgroup $E_{7} \times S U(2)$ is given in [26, 27]. In going to $E_{8(-24)}$ the role played by the subgroup $S L(8, \mathbb{R})$ of $E_{7(7)}$ is played by the subgroup $S U^{*}(8)$ of $E_{7(-25)}$. The quasiconformal groups $E_{8(8)}$ and $E_{8(-24)}$ are isomorphic to the U-duality groups of the maximal $N=16$ supergravity and the $N=4$ exceptional supergravity in three dimensions. Since their action changes the "norm" in the charge-entropy space of the corresponding four dimensional theories they can be interpreted as spectrum generating symmetry groups. The quasiconformal realizations of $E_{8(8)}$ and $E_{8(-24)}$ can be consistently truncated to quasiconformal realizations of other exceptional subgroups.

For the generic Jordan family of $N=2$ MESGT's the quasi-conformal extensions of their U-duality groups $S O(2,1) \times S O(n, 2)$ in $d=4$ are $S O(n+4,4)$, which are isomorphic to their U-duality groups in $d=3$.

\section{The minimal unitary representations of U-duality groups as quasi-conformal groups}

As we saw above the noncompact U-duality groups of four dimensional $N=2$ MESGT's defined by Jordan algebras of degree three can also arise as spectrum generating conformal symmetry groups in five dimensions. These groups all admit positive energy unitary representations which can be constructed using the oscillator method outlined in previous sections. Here I would like to discuss the unitary representations of exceptional groups that one obtains by quantization of their quasiconformal realizations ${ }^{8}$. For $E_{8(8)}$ this was done in [23] and for $E_{8(-24)}$ in [26]. Remarkably, the quantization of the quasiconformal realizations of $E_{8(8)}$ and $E_{8(-24)}$ yield their minimal unitary representations. The concept of a minimal unitary representation of a non-compact group $G$ was first introduced by A. Joseph [28] and is defined as a unitary representation on a Hilbert space of functions depending on the minimal number of coordinates for a given non-compact group. Here we shall summarize the results mainly for $E_{8(8)}$ and indicate how they extend to $E_{8(-24)}$. By truncation one can obtain the minimal unitary realizations of smaller exceptional groups

\footnotetext{
${ }^{8}$ We should note that both the quasiconformal extensions of four dimensional U-duality groups and the isomorphic three dimensional U-duality groups act nonlinearly in their respective dimensions.
} 
as quasiconformal groups as well as unitary realizations of $E_{7(7)}$ and other exceptional subgroups as conformal groups [23, 26, 27].

Since the positive graded generators form an Heisenberg algebra one introduces 28 coordinates $X^{i j}$ and 28 momenta $P_{i j} \equiv X_{i j}$, and one extra real coordinate $y$ to represent the central term. By quantizing

$$
\left[X^{i j}, P_{k l}\right]=\mathrm{i}
$$

we can realize the positive grade generators of $E_{8(8)}$ as

$$
E^{i j}:=y X^{i j}, \quad E_{i j}:=y P_{i j}, \quad E:=\frac{1}{2} y^{2} .
$$

To realize the other generators of $E_{8(8)}$ one introduces a momentum conjugate to the coordinate $y$ representing the central charge of the Heisenberg algebra:

$$
[y, p]=\mathrm{i}
$$

Then the remaining generators are given by

$$
\begin{aligned}
H:= & \frac{1}{2}(y p+p y) \\
F^{i j}:= & -p X^{i j}+2 \mathrm{i} y^{-1}\left[X^{i j}, I_{4}(X, P)\right] \\
= & -4 y^{-1} X^{i k} P_{k l} X^{l j}-\frac{1}{2} y^{-1}\left(X^{i j} P_{k l} X^{k l}+X^{k l} P_{k l} X^{i j}\right) \\
& +\frac{1}{12} y^{-1} \epsilon^{i j k l m n p q} P_{k l} P_{m n} P_{p q}-p X^{i j}, \\
F_{i j}:= & -p P_{i j}+2 \mathrm{i} y^{-1}\left[P_{i j}, I_{4}(X, P)\right] \\
= & 4 y^{-1} P_{i k} X^{k l} P_{l j}+\frac{1}{2} y^{-1}\left(P_{i j} X^{k l} P_{k l}+P_{k l} X^{k l} P_{i j}\right) \\
& -\frac{1}{12} y^{-1} \epsilon_{i j k l m n p q} X^{k l} X^{m n} X^{p q}-p P_{i j}, \\
F:= & \frac{1}{2} p^{2}+2 y^{-2} I_{4}(X, P) \\
G^{i}{ }_{j}:= & 2 X^{i k} P_{k j}+\frac{1}{4} X^{k l} P_{k l} \delta_{j}^{i}, \\
G^{i j k l}:= & -\frac{1}{2} X^{[i j} X^{k l]}+\frac{1}{48} \epsilon^{i j k l m n p q} P_{m n} P_{p q} .
\end{aligned}
$$

The hermiticity of all generators is manifest. Here $I_{4}(X, P)$ is the fourth order differential operator 


$$
\begin{aligned}
I_{4}(X, P):= & -\frac{1}{2}\left(X^{i j} P_{j k} X^{k l} P_{l i}+P_{i j} X^{j k} P_{k l} X^{l i}\right) \\
& +\frac{1}{8}\left(X^{i j} P_{i j} X^{k l} P_{k l}+P_{i j} X^{i j} P_{k l} X^{k l}\right) \\
& -\frac{1}{96} \epsilon^{i j k l m n p q} P_{i j} P_{k l} P_{m n} P_{p q} \\
& -\frac{1}{96} \epsilon_{i j k l m n p q} X^{i j} X^{k l} X^{m n} X^{p q}+\frac{547}{16} .
\end{aligned}
$$

and represents the quartic invariant of $E_{7(7)}$ because

$$
\left[G^{i}{ }_{j}, I_{4}(X, P)\right]=\left[G^{i j k l}, I_{4}(X, P)\right]=0 .
$$

The above unitary realization of $E_{8(8)}$ in terms of position and momentum operators ( Schröedinger picture) can be reformulated in terms of annihilation and creation operators (oscillator realization) ( Bargman-Fock picture) [23]. The transition from the Schrodinger picture to the Bargmann-Fock picture corresponds to going from the $S L(8, \mathbb{R})$ basis to the $S U(8)$ basis of $E_{7(7)}$.

The quadratic Casimir operator of $E_{8(8)}$ reduces to a number for the above realization and one can show that all the higher Casimir operators must also reduce to numbers as required for an irreducible unitary representation. Thus by exponentiating the above generators we obtain the minimal unitary irreducible representation of $E_{8(8)}$ over the Hilbert space of square integrable complex functions in 29 variables.

The $S L(2, \mathbb{R})$ subgroup generated by the grade \pm 2 elements $E, F$ and the dilatation generator $H$ are precisely of the form that arises in conformal quantum mechanics [24]. The quadratic Casimir of this $S L(2, \mathbb{R})$ subgroup is simply

$$
C_{2}\left(S L(2, \mathbb{R})=I_{4}-\frac{3}{16}=\frac{g}{4}-\frac{3}{16}\right.
$$

showing that the role played by the coupling constant $g$ in conformal quantum mechanics is played by the quartic invariant $I_{4}$ of $E_{7(7)}$, which is an $S L(2, \mathbb{R})$ singlet. The role of conformal quantum mechanics in the description of black hole solutions of supergravity was discussed in [25].

In the minimal unitary realization of the other noncompact real form $E_{8(-24)}$ with the maximal compact subgroup $E_{7} \times S U(2)$ given explicitly in [26] the relevant 5-graded decomposition of its Lie algebra $\mathfrak{e}_{8(-24)}$ is with respect to its subalgebra $\mathfrak{e}_{7(-25)} \oplus \mathfrak{s o}(1,1)$

$$
\mathfrak{e}_{8(-24)}=\mathbf{1} \oplus \mathbf{5 6} \oplus\left(\mathfrak{e}_{7(-25)} \oplus \mathfrak{s o}(1,1)\right) \oplus \mathbf{5 6} \oplus \mathbf{1}
$$

The Schrödinger picture for the minimal unitary representation of $E_{8(-24)}$ corresponds to working in the $S U^{*}(8)$ basis of the $E_{7(-25)}$ subgroup. The position and momentum 
operators transform in the $\mathbf{2 8}$ and $\tilde{\mathbf{2 8}}$ of this $S U^{*}(8)$ subgroup and the above formulas for $E_{8(8)}$ carry over to those of $E_{8(-24)}$ with some subtle differences[26]. The Bargmann-Fock picture for the minimal unitary realization of $E_{8(-24)}$ in terms of annihilation and creation operators is obtained by going from the $S U^{*}(8)$ basis to the $S U(6,2)$ basis of the $E_{7(-25)}$ subgroup of $E_{8(-24)}$.

One can obtain the minimal unitary realizations of certain subgroups of $E_{8(8)}$ and $E_{8(-24)}$ by truncating their minimal realizations. However, we should stress that since the minimal realizations of $E_{8(8)}\left[23\right.$ ] and $E_{8(-24)}[26$ ] are nonlinear consistent truncations exist for only certain subgroups. It turns out that the minimal unitary realizations of all lower rank noncompact exceptional groups can be obtained from those of $E_{8(8)}$ and $E_{8(-24)}$ [22, 26]. The relevant subalgebras of $\mathfrak{e}_{8(-24)}$ and $\mathfrak{e}_{8(8)}$ are those that are realized as quasi-conformal algebras, i.e those that have a 5-grading

$$
\mathfrak{g}=\mathfrak{g}^{-2} \oplus \mathfrak{g}^{-1} \oplus \mathfrak{g}^{0} \oplus \mathfrak{g}^{+1} \oplus \mathfrak{g}^{+2}
$$

such that $\mathfrak{g}^{ \pm 2}$ subspaces are one-dimensional and $\mathfrak{g}^{0}=\mathfrak{h} \oplus \Delta$ where $\Delta$ is the generator that determines the 5-grading.. Hence they all have an $\mathfrak{s l}(2, \mathbb{R})$ subalgebra generated by elements of $\mathfrak{g}^{ \pm 2}$ and the generator $\Delta$. For the truncated subalgebra, the quartic invariant $\mathcal{I}_{4}$ will now be that of a subalgebra $\mathfrak{h}$ of $\mathfrak{e}_{7(-25)}$ or of $\mathfrak{e}_{7(7)}$. Furthermore, this subalgebra must act on the grade \pm 1 spaces via symplectic representation. Below we give the main chain of such subalgebras [26]

$$
\mathfrak{h}=\mathfrak{e}_{7(-25)} \supset \mathfrak{s o}^{*}(12) \supset \mathfrak{s u}(3,3) \supset \mathfrak{s p}(6, \mathbb{R}) \supset \oplus_{1}^{3} \mathfrak{s p}(2, \mathbb{R}) \supset \mathfrak{s p}(2, \mathbb{R}) \supset \mathfrak{u}(1)
$$

Corresponding quasi-conformal subalgebras read as follows

$$
\mathfrak{g}=\mathfrak{e}_{8(-24)} \supset \mathfrak{e}_{7(-5)} \supset \mathfrak{e}_{6(2)} \supset \mathfrak{f}_{4(4)} \supset \mathfrak{s o}(4,4) \supset \mathfrak{g}_{2(2)} \supset \mathfrak{s u}(2,1)
$$

The corresponding chains for the other real form $\mathfrak{e}_{8(8)}$ are

$$
\begin{aligned}
& \mathfrak{h}=\mathfrak{e}_{7(7)} \supset \mathfrak{s o}(6,6) \supset \mathfrak{s l}\left(6, \mathbb{R} \supset \mathfrak{s p}(6, \mathbb{R}) \supset \oplus_{1}^{3} \mathfrak{s p}(2, \mathbb{R}) \supset \mathfrak{s p}(2, \mathbb{R}) \supset \mathfrak{u}(1)\right. \\
& \mathfrak{g}=\mathfrak{e}_{8(8)} \supset \mathfrak{e}_{7(7)} \supset \mathfrak{e}_{6(6)} \supset \mathfrak{f}_{4(4)} \supset \mathfrak{s o}(4,4) \supset \mathfrak{g}_{2(2)} \supset \mathfrak{s u}(2,1)
\end{aligned}
$$

The minimal unitary realizations of $\mathfrak{e}_{8(8)}$ and of $\mathfrak{e}_{8(-24)}$ can also be consistently truncated to unitary realizations of certain subalgebras that act as regular conformal algebras with a 3 -grading. For $\mathfrak{e}_{8(8)}$ we have the following chain of consistent truncations to conformal subalgebras conf:

$$
\mathfrak{c o n f}=\mathfrak{e}_{7(7)} \supset \mathfrak{s o}(6,6) \supset \mathfrak{s l}\left(6, \mathbb{R} \supset \mathfrak{s p}(6, \mathbb{R}) \supset \oplus_{1}^{3} \mathfrak{s p}(2, \mathbb{R}) \supset \mathfrak{s p}(2, \mathbb{R})\right.
$$

The corresponding chain of consistent truncations to conformal subalgebras for $\mathfrak{e}_{8(-24)}$ is

$$
\mathfrak{c o n \mathfrak { f }}=\mathfrak{e}_{7(-25)} \supset \mathfrak{s o}^{*}(12) \supset \mathfrak{s u}(3,3) \supset \mathfrak{s p}(6, \mathbb{R}) \supset \oplus_{1}^{3} \mathfrak{s p}(2, \mathbb{R}) \supset \mathfrak{s p}(2, \mathbb{R})
$$




\section{Concluding Remarks}

The minimal unitary realizations of $E_{8(8)}$ and $E_{8(-24)}$ and their subgroups given in [23, 26] can be extended to all noncompact groups and supergroups $[27]^{9}$ and formulated in a unified manner. This unified construction follows closely the formalism of unified construction of nonlinear superconformal and quasi-superconformal algebras in two dimensions [29]. The realization of the distinguished $S L(2, \mathbb{R})$ subgroup generated by the grade \pm 2 elements in the unified construction is always of the form that arises in conformal or superconformal quantum mechanics. The coherent state formulation of the minimal unitary representations of noncompact groups and supergroups analogous to those of conformal groups is currently under investigation.

As I stated at the beginning of my talk, the discrete subgroups $E_{(11-d)(11-d)}(\mathbb{Z})$ of the U-duality groups of M-theory toroidally compactified to d dimensions are expected to be non-perturbative symmetries of M-theory [4]. One expects similarly that certain discrete subgroups of the U-duality groups of matter coupled supergravity theories obtained by other compactifications of M/superstring theory to be non-perturbative symmetries in the respective dimensions. The major goal of the work summarized in this talk is to find the unitary realizations of these discrete subgroups and understand how the spectra of compactified M/Superstring theory fit into these representations.

Acknowledgement: I would like to thank the organizers of the XIXth Max Born Symposium in Wroclaw for their kind hospitality. The work presented here was done mostly in collaboration with Kilian Koepsell, Hermann Nicolai and Olexander Pavlyk, which I would like to acknowledge with pleasure. This work was supported in part by the National Science Foundation under grant number PHY-0245337. Any opinions, findings and conclusions or recomendations expressed in this material are those of the author and do not necessarily reflect the views of the National Science Foundation.

\section{References}

[1] E. Cremmer, B. Julia and J. Scherk, "Supergravity Theory in 11 Dimensions", Phys. Lett. , B76, 409 (1978).

[2] E. Witten, “ String Theory Dynamics in various Dimensions", Nucl. Phys. B443 , 85 (1995).

\footnotetext{
${ }^{9}$ For $S L(2, \mathbb{R})$ the minimal realization reduces to the conformal realization.
} 
[3] B Julia, “Group Disintegrations”, in: Superspace and Supergravity, eds. S.W.Hawking and M. Rocek, Cambridge University Press, 1981.

[4] C. Hull and P.K. Townsend, "Unity of Superstring Dualities”, Nucl.Phys. B438, 109 ( 1995).

[5] M. Günaydin, G. Sierra and P.K. Townsend, Nucl. Phys. B242 (1984), 244 ; Phys. Lett. 133B (1983) 72.

[6] M. Günaydin and M. Zagermann," Unified Maxwell-Einstein and Yang-Mills-Einstein supergravity theories in five dimensions," JHEP 0307, 023 (2003)

[7] S. Ferrara and R. Kallosh, Phys. Rev. D54 (1996) 5344; hep-th/9603090. For further references on the subject see [8, 9].

[8] See S. Ferrara and J. Maldacena, "Branes, central charges and U-duality invariant BPS conditions", Class.Quant.Grav. 15749 ( 1998) and the references therein.

[9] S. Ferrara and M. Gunaydin, "Orbits of exceptional groups, duality and BPS states in string theory," Int. J. Mod. Phys. A13, 2075 (1998)

[10] R. Kallosh and B. Kol, Phys. Rev. D53 (1996) 1525; hep-th/9602014 For further references on the subject see [8, 9].

[11] M. Günaydin, Nuovo Cimento 29A (1975) 467.

[12] M. Günaydin, Ann. Israel Physical Society 3 (1980) 279.

[13] M. Günaydin, in "Elementary Particles and the Universe: Essays in Honor of Murray Gell-Mann", ed. by J.H. Schwarz ( Cambridge University Press, 1991); M. Günaydin, “On An Exceptional Nonassociative Superspace," J. Math. Phys. 31, 1776 (1990).

[14] M. Günaydin, "Generalized conformal and superconformal group actions and Jordan algebras," Mod. Phys. Lett. A8, 1407 (1993) [hep-th/9301050].

[15] M. Günaydin and C. Saclioglu, Comm. Math. Phys., 87 (1982) 159; Phys. Lett. B108 (1982) 180.

[16] M. Günaydin," AdS/CFT Dualities and the Unitary Representations of Non-compact Groups and Supergroups: Wigner versus Dirac", invited talk in the proceedings of the VIth International Wigner Symposium (Istanbul, 1999), hhep-th/0005168], ed. by M. Arik, Bogazici University Press, 2002, pp. 55-69. 
[17] M. Günaydin, D. Minic and M. Zagermann, hep-th/9810226, Nucl. Phys. B544 (1999) 737-758; hep-th/9806042, Nucl. Phys. B534 (1998) 96-120.

[18] M. Günaydin and S. Takemae, "Unitary Supermultiplets of $O S p\left(8^{*} \mid 4\right)$ and the $A d S_{7} / C F T_{6}$ Duality", Nucl. Phys. B 578 (2000) 405-448 [ arXiv: hep-th/9910110 ] ; S. Fernando, M. Günaydin and S. Takemae, "Supercoherent states of $\operatorname{OSp}\left(8^{*} \mid 2 \mathrm{~N}\right)$, conformal superfields and the AdS(7)/CFT(6) duality," Nucl. Phys. B 628, 79 (2002) [arXiv:hep-th/0106161].

[19] M. Günaydin, in preparation.

[20] I. Bars and M. Günaydin, Comm. Math. Phys., 91 (1983) 21.

[21] G. Mack and A. Salam, Ann. Phys. 53 (1969) 174; G. Mack, Comm. Math. Phys. 55 (1977) 1.

[22] M. Gunaydin, K. Koepsell and H. Nicolai, "Conformal and quasiconformal realizations of exceptional Lie groups," Commun. Math. Phys. 221, 57 (2001)

[23] M. Gunaydin, K. Koepsell and H. Nicolai, "The Minimal Unitary Representation of $E_{8(8)}, "$ Adv. Theor. Math. Phys. 5, 923 (2002)

[24] V. de Alfaro, S. Fubini, G. Furlan. Conformal invariance in quantum mechanics. Nuovo Cim. A34, 569 (1976)

[25] P. Claus, M. Derix, R. Kallosh, J. Kumar, P. Townsend, A. Van Proeyen. Black holes and superconformal mechanics. Phys. Rev. Lett. 81, 4553 (1998)

[26] M. Gunaydin and O. Pavlyk, " Minimal unitary realizations of exceptional Uduality groups and their subgroups as quasiconformal groups", JHEPO1 ( 2005) 019 [hep-th/0409272].

[27] M. Gunaydin and O. Pavlyk, in preparation.

[28] A. Joseph, "Minimal realizations and spectrum generating algebras", Commun. Math. Phys. 36, 325 (1974); "The minimal orbit in a simple Lie algebra and its associated maximal ideal", Ann. Sci. Ec. Norm. Super, IV. Ser. 9, 1 (1976). For further references on the minimal unitary representations of noncompact exceptional groups see [23].

[29] B. Bina and M. Günaydin. Real forms of non-linear superconformal and quasisuperconformal algebras and their unified realization. Nucl. Phys. B502, 713 (1997). 